Kaitai shinsho, The single most Famous Japanese Book of Medecine and Geka sōden, An early very Important Manuscript on Surgery, G. LUKACS

\title{
Annick Horiuchi
}

\section{(2) OpenEdition}

Édition électronique

URL : https://journals.openedition.org/cipango/437

DOI : $10.4000 /$ cipango.437

ISSN : 2260-7706

Éditeur

INALCO

\section{Édition imprimée}

Date de publication : 1 janvier 2008

Pagination : 254-258

ISBN : 978-2-85831-177-4

ISSN : $1164-5857$

Référence électronique

Annick Horiuchi, « KAITAI SHINSHO, the SINgle MOST FAMOUS JAPANESE BOOK OF MEDECINE AND GEKA SŌDEN, AN EARLY VERY IMPORTANT MANUSCRIPT ON SURgERY, G. LUKACS 》, Cipango [En ligne], 15 | 2008, document 6, mis en ligne le 13 novembre 2011, consulté le 30 juin 2021. URL : http:// journals.openedition.org/cipango/437 ; DOI : https://doi.org/10.4000/cipango.437

Ce document a été généré automatiquement le 30 juin 2021.

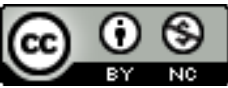

Cipango est mis à disposition selon les termes de la Licence Creative Commons Attribution - Pas d'Utilisation Commerciale 4.0 International. 


\title{
Kaitai shinsho, The single most Famous Japanese Book of Medecine and Geka sōden, An early very Important Manuscript on Surgery, G. LUKACS
}

\author{
Annick Horiuchi
}

\section{RÉFÉRENCE}

Kaitai shinsho, The single most Famous Japanese Book of Medecine and Geka sōden, An early very Important Manuscript on Surgery, de G. LUKACS, Hes \& De Graaf Publishers, BV, PaysBas, 2008, 286 p. ISBN 978-90-6194-499-7

1 À l'instar de l'historien Jacques Proust, grand spécialiste du siècle des lumières, qui s'était tourné vers le Japon au terme de sa carrière et nous a laissé une magnifique réflexion sur la manière dont les savoirs occidentaux ont trouvé leur chemin au Japon ${ }^{1}$, l'ancien directeur de recherches du laboratoire de chimie des substances naturelles du CNRS Gabor Lukacs poursuit depuis sept ans une recherche assidue et fructueuse sur les ouvrages qui ont joué un rôle important dans la diffusion des sciences occidentales au Japon. Dans le présent ouvrage, il nous présente les résultats de ses recherches sur deux ouvrages d'anatomie japonais de l'époque d'Edo: le Kaitai shinsho 解体新書 (Le Nouveau livre d'anatomie), xylographié en 1774, et le Geka sōden 外科宗伝 ${ }^{2}$, manuscrit achevé en 1706. La chronologie aurait voulu que le second ouvrage soit abordé avant le premier mais l'objectif de G. Lukacs n'est pas de survoler l'histoire de la médecine chinoise : il pose sur les ouvrages d'anatomie japonais un regard de scientifique et d'expert en bibliographie, et, en ce faisant, il ouvre des pistes de réflexion qui ne manqueront pas de stimuler les historiens.

2 Bien que G. Lukacs ne prétend jamais au titre de japonologue pas plus qu'à celui d'historien de la médecine du Japon, il n'en a pas moins accumulé dans ce domaine une 
connaissance impressionnante. Son ouvrage apparait comme l'un des mieux documentés existant à ce jour, et sa bibliographie regroupée en fin de volume est des plus complètes, s'agissant des études en langues occidentales. L'auteur salue au passage les travaux de Wolfgang Michel et plus particulièrement de Mieko Macé qui lui ont permis de saisir d'au plus près la pensée médicale japonaise. On regrettera seulement qu'il ne mentionne à aucun moment les ressources électroniques qui constituent désormais un accès majeur aux sources premières.

3 G. Lukacs ne s'attarde pas sur les grandes étapes de l'histoire de la médecine au Japon, se contentant de renvoyer à des références désormais classiques (mentionnées dans les notes p.19). Il s'intéresse d'emblée au célèbre Kaitai shinsho que les historiens considèrent généralement et non sans raison comme l'ouvrage qui marque l'entrée de la médecine japonaise dans l'ère de la modernité. D'après le récit rapporté par Sugita Genpaku dans le Rangaku kotohajime (Commencement des études hollandaises, 1815), ce projet de traduction collectif aurait vu le jour à la suite d'une dissection à laquelle Sugita et deux de ses amis médecins auraient assisté en 1771. D'après ce récit, qu'il convient de traiter avec prudence car il a été écrit plus de quarante ans après les faits, cette expérience aurait convaincu le groupe de médecins de la qualité des illustrations d'un ouvrage d'anatomie dont ils étaient en possession: le Ontleedkundige Tafelen (les Tables anatomiques ; 1734) du médecin allemand Johann Adam Kulmus.

G. Lukacs va montrer avec élégance qu'en dépit des très nombreuses études consacrées à cet ouvrage au Japon, il restait encore des points à éclaircir, notamment tout ce qui touche à son processus d'élaboration et à son impact sur ses contemporains. Il commence d'abord par s'intéresser à l'ouvrage source que sont les Tables anatomiques de Kulmus (1689-1745), un ouvrage d'anatomie allemand destiné à des débutants, qui rencontra un énorme succès public comme en attestent les vingt-deux éditions répertoriées (en quatre langues). L'auteur examine, en bon bibliographe, les différents exemplaires des différentes éditions et s'attarde plus particulièrement sur l'unique édition hollandaise parvenue au Japon et ayant été à l'origine de la traduction japonaise. Il met en relief les caractéristiques du livre que Sugita et ses acolytes avaient entre les mains, sa composition, ses illustrations, ses notes en bas de page et en traduit quelques passages. A cette occasion, Lukacs corrige certaines interprétations proposées par les spécialistes japonais, par méconnaissance des techniques d'impression en usage en Occident. En s'attardant ainsi sur l'ouvrage de Kulmus, l'auteur apporte cet éclairage si fondamental mais trop souvent négligé qui permet de resituer ces ouvrages de science occidentaux dans leur propre contexte et ce faisant de mieux comprendre pourquoi ils ont trouvé leur chemin vers le Japon à ce moment précis et pourquoi ils ont retenu l'attention des médecins japonais.

5 C'est en faisant appel à ses compétences en matière de livres anciens qu'il entreprend sa grande enquête sur les nombreux exemplaires du Kaitai shinsho conservés dans les bibliothèques du monde entier, nous faisant découvrir des subtilités jusqu'alors insoupçonnées sur le travail de composition des médecins japonais, sur les différentes dates de publication de ce traité d'anatomie, ainsi que sur l'accueil qui a été réservé par les contemporains. Les trouvailles sont nombreuses et même lorsqu'il ne parvient pas à lever le mystère (c'est le cas notamment de l'origine de l'exemplaire du Kaitai shinsho acquis par la Bibliothèque nationale en 1839), les interrogations qu'il soulève en chemin sont riches d'enseignements. 
6 Une contribution importante de G. Lukacs réside dans le recensement effectué dans les grandes bibliothèques du monde des exemplaires du Kaitai shinsho, exemplaires qu'il a pris la peine d'examiner un à un. Il dit en avoir consulté 55, mais plusieurs exemplaires lui ont été signalés une fois le livre rédigé et il n'ignore pas qu'il faut y ajouter encore plus d'une quarantaine hors d'atteinte car en possession de collectionneurs privés. A travers l'observation de ces ouvrages, il entreprend de les dater, une tâche qui s'avère d'une difficulté redoutable du fait que la date inscrite sur le colophon, reproduite à l'identique dans le cas du Kaitai shinsho sur tous les exemplaires, ne correspond pas nécessairement à la date d'édition réelle de l'ouvrage. G. Lukacs déploie des trésors d'ingéniosité pour parvenir à distinguer des catégories au sein de ces exemplaires en utilisant par exemple le changement d'adresse du libraire Suwaraya Ichibee ou au moyen des pages de publicité insérées en fin de volume. Il nous renseigne au passage sur ce libraire, éditeur du fameux Kaikoku heidan (De la défense d'un pays maritime) de Hayashi Shihei, dont on sait qu'il fut frappé d'interdit en 1791. G. Lukacs nous offre ici un bel exemple de la nécessité d'examiner de près cet objet qu'est le livre dès lors que l'on s'intéresse à l'histoire de la diffusion des savoirs.

7 D'autres mini-enquêtes attirent l'attention comme celle menée autour du célèbre frontispice du Kaitai shinsho (chap. 3, partie I), dont on sait à partir d'études antérieures, qu'il s'agit d'une reproduction en format réduit du frontispice du Vivae Imagines Corporis Humani de Juan de Valverde. G. Lukacs montre qu'un examen attentif de l'image permet d'éclaircir des mystères qu'elle recèle encore et de dévoiler les raisons pour lesquelles le groupe de médecins et le dessinateur Odano Naotake ont pu être amenés à retoucher l'image de cette façon.

8 Le regard distant et critique que notre auteur observe vis-à-vis des discours japonais qui entourent le Kaitai shinsho et sa connaissance de l'histoire de l'anatomie en Occident le conduisent à cette conclusion surprenante mais convaincante que, compte tenu de la difficulté pour un œil inexpérimenté de distinguer les différents organes au cours d'une dissection et de la méconnaissance de la science anatomique des médecins réunis autour de Sugita en 1771, l'expérience de la dissection n'a sans doute pas été déterminante dans leur décision d'adopter le paradigme de la médecine occidentale. Autrement dit, ces savants ne demandaient qu'à être convaincus ...

9 Mais l'apport le plus important de cette étude de G. Lukacs réside sans doute dans le travail réalisé sur les annotations du Kaitai shinsho. L'auteur dit s'être inspiré du travail de 0 . Gingerich réalisé à partir du recensement des différents exemplaires du De Revolutionibus de Copernic ${ }^{3}$ conservés dans le monde et ayant pour objet d'étudier la réception qui fut réservée à cet ouvrage majeur de la science en Europe. La recherche menée par Lukacs permet d'établir de la même manière que le Kaitai shinsho a eu un impact considérable sur ses contemporains. Les exemplaires richement annotés sont nombreux. L'auteur s'est plus particulièrement intéressé aux annotations laissées par le médecin de Kyoto, Koishi Genshun 小石元俊 (1743-1808), réputé pour sa contribution dans le domaine de l'anatomie et des dissections. Son étude lève le voile sur des pratiques de lecture jusque là totalement ignorées par les historiens japonais. Il apparaît ainsi que de nombreuses pratiques d'annotation attestées en Occident sont également présentes au Japon et que, s'agissant du Kaitai shinsho, l'ouvrage a suscité, plus que tout autre ouvrage de médecine, de copieuses annotations. Certains exemplaires comportent ainsi plusieurs strates d'annotations et Lukacs a également pu établir que les annotations de Koishi Genshun avaient fait été recopiées par ses élèves 
sur leurs propres exemplaires. L'auteur ne se contente pas d'attirer l'attention sur ces annotations. Il en propose une méthode d'analyse qu'il applique sur certains passages du livre. Il examine notamment le volume d'annotations, leur emplacement, et leur nature: si elles portent sur des questions d'érudition ou des questions proprement médicales. Lukacs ouvre ainsi des pistes de recherche passionnantes qui devraient inspirer d'autres chercheurs après lui et modifier définitivement le regard porté sur les ouvrages anciens annotés.

10 L'auteur réserve la deuxième partie du livre à l'ouvrage de chirurgie de Narabayashi Chinzan (1648-1711), intitulé Geka sōden (manuscrit daté de 1706), dont il a pu se procurer personnellement une copie manuscrite contenant des illustrations très soignées, en couleur. Narabayashi Chinzan est issu d'une famille d'interprètes au service des Hollandais de Nagasaki. Là encore, Lukacs commence par s'intéresser à la situation de la chirurgie en Europe au XVI ${ }^{e}$ et $\mathrm{XVII}^{\mathrm{e}}$ siècles et plus particulièrement aux ouvrages hollandais par lesquels s'est effectuée la transmission de la chirurgie occidentale au Japon. Il retient plus particulièrement les œuvres d'Ambroise Paré (original paru en 1575) et l'Armamentarium Chirugicum (1655) du médecin allemand Scultetus (Schultes). Il se tourne ensuite vers les manuscrits du Geka sōden et l'étude est cette fois plus complexe du fait qu'il ne s'agit pas d'un ouvrage xylographié et qu'il contient des illustrations en couleur. La conclusion de son étude est que le Geka sōden est le fruit d'une élaboration beaucoup plus sophistiquée que ne l'ont imaginé les historiens et que s'agissant des superbes illustrations contenues dans la partie intitulée Kinsō tetsuboku zu 金瘡跌撲図, trois sources hollandaises auraient été utilisées dont le Scultetus. Quant aux textes qui les accompagnent, leur filiation avec les textes hollandais est beaucoup moins directe. Bref, le livre de Narabayashi, qui conserve encore bien des mystères, révèle une connaissance déjà très poussée de la médecine hollandaise à Nagasaki au début du XvIII ${ }^{e}$ siècle. Elle révèle aussi une grande curiosité à l'égard des nouveautés comme la chirurgie du crâne, un intérêt prononcé pour les instruments de chirurgie (reproduits en détail), ainsi qu'une attitude pragmatique qui amène à compléter le savoir venu d'Occident par des connaissances puisées aux sources chinoises, notamment dans le Geka seisō 外科正宗 (Waike Zhengzong; Le véritable enseignement de la chirurgie ; 1617), plusieurs fois édité en Chine, sous les Ming et les Qing.

11 Disons pour finir que, Lukacs s'est manifestement amusé à faire de son livre un objet agréable à feuilleter et à lire. De nombreuses illustrations en couleur appuient son argumentation et les notes sont délicatement insérées dans la marge ou en bas de page, comme une sorte de clin d'œil aux savants des temps anciens qui griffonnaient dans tous les recoins. Le livre pourra paraître très spécialisé au premier abord mais la passion de l'auteur pour la médecine, les livres et la culture japonaise est communicative. Le livre devrait ainsi intéresser le non-spécialiste comme l'historien du livre ou l'historien de la médecine qui y trouveront toutes les informations utiles pour pousser plus loin la recherche. 


\section{NOTES}

1. Jacques Proust, L'Europe au prisme du Japon, XVI-XVIII esiècle, Entre humanisme, contre-réforme et lumières, Albin Michel, 1997.

2. Titre non traduit dans le livre et qui correspond à: «Enseignement authentique de chirurgie ".

3. Gingerich, Owen, An Annotated Census of Copernicus's De Revolutionibus, Nürnberg, 1543, and Basel 1566, (Leiden, Boston and Köln, Brill, 2002). 